\title{
The longitudinal association between the use of antihypertensive medications and 24-hour sleep in nursing homes: results from the randomized controlled COSMOS trial
}

\author{
Elisabeth Flo-Groeneboom ${ }^{1,2^{*}}$ (D), Tony Elvegaard ${ }^{1}$, Christine Gulla ${ }^{1,3}$ and Bettina S Husebo ${ }^{1,4}$
}

\begin{abstract}
Background: Antihypertensive medication use and sleep problems are highly prevalent in nursing home patients. While it is hypothesized that blood pressure and antihypertensive medication use can affect sleep, this has not been investigated in depth in this population. Alongside a multicomponent intervention including a systematic medication review, we aimed to investigate the longitudinal association between antihypertensive medication use, blood pressure and day- and night-time sleep over 4 months.

Methods: This study was based on secondary analyses from the multicomponent cluster randomized controlled COSMOS trial, in which the acronym denotes the intervention: COmmuncation, Systematic pain assessment and treatment, Medication review, Organization of activities and Safety. We included baseline and 4-month follow-up data from a subgroup of nursing home patients who wore actigraphs $(n=107)$. The subgroup had different levels of blood pressure, from low $(<120)$ to high $(\geq 141)$. Assessments included blood pressure, antihypertensive medication use, and sleep parameters as assessed by actigraphy.

Results: We found a significant reduction in total sleep time at month four in the intervention group compared to the control group. When analysing the control group alone, we found a significant association between antihypertensive medication use and increased daytime sleep. We also found negative associations between blood pressure, antihypertensive medication use and sleep onset latency in the control group.

Conclusions: Our results suggest a correlation between excessive daytime sleep and antihypertensive medication use. These findings should be followed up with further research, and with clinical caution, as antihypertensive medications are frequently used in nursing homes, and sleep problems may be especially detrimental for this population.
\end{abstract}

Trial registration: The trial is registered at clinicaltrials.gov (NCT02238652).

Keywords: Sleep, Actigraphy, Blood pressure, Antihypertensives, Antihypertensive medication use

\footnotetext{
* Correspondence: Elisabeth.flo@uib.no

${ }^{1}$ Centre for Elderly and Nursing Home Medicine, Department of Global Public Health and Primary Care, University of Bergen, Bergen, Norway

${ }^{2}$ Department of Clinical Psychology, University of Bergen, Postboks 7807, 5020 Bergen, Norway

Full list of author information is available at the end of the article
}

C C The Author(s). 2021 Open Access This article is licensed under a Creative Commons Attribution 4.0 International License, which permits use, sharing, adaptation, distribution and reproduction in any medium or format, as long as you give appropriate credit to the original author(s) and the source, provide a link to the Creative Commons licence, and indicate if changes were made. The images or other third party material in this article are included in the article's Creative Commons licence, unless indicated otherwise in a credit line to the material. If material is not included in the article's Creative Commons licence and your intended use is not permitted by statutory regulation or exceeds the permitted use, you will need to obtain permission directly from the copyright holder. To view a copy of this licence, visit http://creativecommons.org/licenses/by/4.0/ The Creative Commons Public Domain Dedication waiver (http://creativecommons.org/publicdomain/zero/1.0/) applies to the data made available in this article, unless otherwise stated in a credit line to the data. 


\section{Background}

Sleep problems, excessive sleepiness and daytime sleep are commonly observed in the nursing home population $[1,2]$. A hallmark study by Jacobs et al., demonstrated that nursing home patients with dementia were neither awake nor asleep for a full hour throughout a 24-h period [3]. Although increased daytime sleep may be considered an adaptive response to impaired night-time sleep, it is well-known that naps negatively impacts sleep at night [1]. This pattern of daytime sleep and nighttime wake-periods is common in nursing home patients and in people with dementia [4].

Today's nursing home patients are frail and multimorbid; $80 \%$ have dementia, and almost half the patients have cardiovascular diseases such as hypertension, heart failure, and atrial fibrillation [5]. There is inconclusive research regarding the potential benefits or unwanted side effects and risks of antihypertensive medication use in nursing home patients and people with dementia [6]. While some argue that treatment is beneficial regardless of age [7, 8], others are more concerned, highlighting risks of side effects and adverse effects [9, 10]. In patients with dementia, antihypertensive treatment is associated with orthostatic hypotension, falls, and increased anticholinergic burden [11].

Although sleepiness and nightmares are listed as common side effects in antihypertensive medications, no studies have investigated the association between such medications and night- and daytime sleep in a nursing home population or in people with dementia. Meanwhile, effects in the central nervous system have been associated with centrally acting antihypertensive medications. Early studies demonstrated that beta-blockers affect sleep architecture and maintenance, with increased awakenings associated with lipophilic beta-blockers, including propranolol [12]. In an early study by Gislason et al., including 4064 Swedish men, a significant risk of excessive daytime sleepiness was associated with hypertension, but not with the use of beta-blockers [13]. A study by Nicholson et al. found an increased risk of sleepiness in six adult males after antihypertensive treatment [14]. None of these studies included older individuals, or people with dementia. In addition, a recent review of the antihypertensive treatment in people with dementia did not identify any study investigating sleep disturbances other than sleep apnea in relation to the use of antihypertensive medication [15].

There has been demonstrated a link between cardiovascular disease and disturbed sleep in elderly populations [13, 16-18]. One Japanese prospective cohort study investigated the association between sleep and nocturnal blood pressure in 107 institutionalized people with dementia, while controlling for antihypertensive treatment [19]. The authors found an association between night-time sleep problems and impaired reduction in blood pressure, though they did not identify any moderating effects of antihypertensive medication use. In a study by Kostis and colleagues, they found a negative effect of antihypertensive medication use (propranolol) on total sleep time and sleep maintenance (waking after sleep onset) in adults with mild hypertension, but found no effects in an older population (60-78 years) [20].

Ageing is related to several changes in sleep, including reduced total sleep time and increased sleep fragmentation e.g., waking during the main sleep period. Consequently, it is also more common to observe daytime napping [21, 22]. These changes in sleep are complex and multicausal. They may partly be due to neurological changes, such as loss of cells in the "master clock", superchiasmatic nuclei. Also, several conditions that are increasingly common in old age include symptoms that can disrupt sleep. This includes congestive heart failure and coronary artery disease [23, 24]. Sleep problems may share physiological mechanisms with high blood pressure. Problems with falling asleep, sleep fragmentation and waking up early have been related to a state of hyperarousal [25]. Having these sleep problems in combination with hyperarousal has been associated with a significant risk of hypertension [26]. In addition, the use of beta-blockers may inhibit pineal gland activity, resulting in suppressed levels of the melatonin during the night, which may negatively impact sleep [27].

In a recent study where we implemented a systematic medication review in nursing homes, we found that both nursing home patients with low and normal blood pressure (i.e., a systolic and diastolic blood pressure below $160 \mathrm{mmHg}$ and $90 \mathrm{mmHg}$ ) used a median of one antihypertensive medication. Those with high blood pressure (a systolic and diastolic blood pressure above $160 \mathrm{mmHg}$ and $90 \mathrm{mmHg}$ ) had a median of two antihypertensive medications. The medication review reduced the use of antihypertensive medications, leading to a temporarily increase in the systolic blood pressure. Meanwhile, by follow-up at month nine, the blood pressure had reached initial levels [28, 29].

Cardiovascular disease and antihypertensive medications may cause both day- and night-time symptoms affecting sleep. Consequently, it is necessary to investigate sleep parameters throughout a 24-h period. No study has investigated this association using 24-h, objective sleep assessment by actigraphy. This current study aimed to investigate the effect of a multicomponent intervention, including a systematic medication review, on day- and night-time sleep in a large nursing home population. In particular, we investigated the association between antihypertensive medication use, blood pressure and day- and night-time sleep at baseline and at 4 months follow-up. 


\section{Methods}

The current study was based on secondary analyses from the cluster randomized controlled COSMOS trial (cRCT). This was a multicomponent intervention trial, where the COSMOS acronym represents the intervention components: COmmunication, Systematic pain assessment and treatment, Medication review, Organization of activities, and Safety. The trial was conducted between May 2014 and December 2015. The published protocol describes the trial design, intervention, and sample size analyses in detail [30]. Related articles describe different aspects of the trial for instance the effect of the intervention on quality of life and neuropsychiatric symptoms [29], the effect of communication [31, 32], and the effect of the medication review $[28,33]$.

\section{Study design and participants}

The COMSOS trial was an cRCT, lasting for four months, with data assessments at baseline, month four and a nine-month follow-up. We invited 765 patients from 72 units in 37 nursing homes from eight municipalities in Western and Eastern Norway to participate. For the actigraphy subproject, which provides data for the present study, 107 patients from both the intervention and control group were randomly selected from 19 nursing home units in four municipalities. Only nursing home unites with long-term care patients were included. Exclusion criteria were life expectancy $<6$ months, patients $<65$ years, and schizophrenia. In the present study, we used baseline and 4-month data, as there was no actigraphic measurements in either control or intervention group at the nine-month follow-up. In addition, patients suffering from any form of chronic upper body movement disorder or paralysis, and patients with less than 5 days and nights of actigraphy recordings, were excluded from the actigraphy subproject.

\section{Intervention}

The multicomponent COSMOS intervention was implemented by a two-day educational seminar for all intervention units. The educational seminar included training and lectures in the multicomponent COSMOS intervention: Communication in the form of Advanced Care Planning, systematic pain assessment and treatment, medication review, organization of activities and safety. These units sent at least two nurses to the seminar who became that unit's COSMOS ambassadors. In addition, the nursing home managers, physicians, and pharmacist were invited. The education program covered researchbased knowledge about communication and advance care planning, pain assessment and treatment, multidisciplinary medication review, and organization of activities. After the seminar, the COSMOS ambassadors trained the rest of the staff in their units [34]. For the medication review, the COSMOS ambassadors and physicians each received their own written material, preparing them for the multidisciplinary medication review session together with two researchers (BSH and CG). Prior to the medication review, nurses, alongside the researchers assessed the patients with relevant clinical tools, and extracted the patients' medication list, blood results, and diagnoses from the medical records. The physicians received a short description of the assessment tools used in the medication review, the Norwegian guidelines for medication reviews $[35,36]$, the STOPP/ START version 2 criteria potentially inappropriate prescribing in older people [37], and an anticholinergic medications list [38].

\section{Outcome measures}

Sleep was assessed with actigraphy, using the Philips Actiwatch Spectrum, which was worn on the patients' dominant or mobile wrist continuously for $24 \mathrm{~h}$ throughout 7 consecutive days at baseline and month 4 $[39,40]$. The actigraphs were placed on the dominant/ mobile wrist to increase movement detection in this immobile population. The data was analysed with the Respironics Actiware 5 software, yielding the following standard sleep parameters: minutes of daytime sleep, minutes of sleep onset latency (time from going to bed until falling asleep), minutes of wake after sleep onset, minutes of early morning awakening (defined as minutes from waking up, until helped out of bed) and minutes of total sleep time. All variables were calculated as mean minutes per day/night for all patients with at least five valid days of actigraphy recording. Nursing home staff received both verbal and written instruction to push the event button on the actigraph at bed and rise times (light off in the night/light on in the morning).

In the sleep scoring protocol, rest intervals were set using a standardized hierarchical approach based on: (1) event markers, (2) light and activity data, and (3) light or activity data. Inter-scorer reliability was ensured by comparing 30 actigraphy recordings, scored by two independent scorers, in terms of total time in bed and total sleep time. Participants had to complete at least fivenight recordings to be included. Sleep/wake status was scored for each one-minute epoch using the Actiware 6 software, with the sensitivity set to medium.

Medications registered in the patients' medical records were coded according to Anatomical Therapeutic Chemical Index (ATC) classes [41]. The number of antihypertensive medications summed for each patient derived from the following five medication classes: C03C High Ceiling Diuretics (loop-diuretics), C07A Beta-blockers, C09A Plain Angiotensin-Converting-Enzyme Inhibitors, C09C Plain Angiotensin II Antagonists, and C08C Calcium Channel Blockers with mainly vascular effect. 
Blood pressure (measured in $\mathrm{mmHg}$ ) and pulse was assessed in adherence with local procedure. Diagnoses were obtained from the participants" medical records.

Functioning in terms of cognitive function and activities of daily living, was assessed using the Mini Mental State Examiner (MMSE) and the Lawton and Brody Selfmaintenance Scale. The MMSE assesses the level of cognitive impairment. Scores range from 0 to 30, with the following recommended cut-offs: $0-11=$ severe dementia, $12-17=$ moderate dementia,$\quad 18-23=$ mild dementia, and 24-30=no [42]. the Lawton and Brody Self-maintenance Scale includes six items (composite score range $0-30$ ), where a lower value indicates better functioning and independence [43].

\section{Sample size analysis}

The COSMOS trial's power analysis was based on the primary outcome, quality of life. Based on the change expected, the sample size was estimated to 520 participants in total, factoring in cluster design and drop out. No posteriori analysis was performed for secondary analyses.

\section{Randomization and blinding}

The included nursing home units were randomized to intervention groups or control groups (standard care) per participating municipality. Each unit was defined as a cluster and was randomized with a random number sequence in SPSS 18. The randomization was completed as a constrained complete list randomization stratified on 33 participating sites to ensure almost equal matched distribution to geographic and monetary status. This was a single-blinded study where the nursing homes were naïve to their allocation. When using interventions like staff education, with a clear focus on implementation, double-blinding is not feasible. Indeed, the single-blind design is described as most appropriate.

\section{Statistical analyses}

Both the intervention and control group were included in descriptive analyses and baseline analyses, as well as in the analyses of the intervention effect. Because the intervention group received a multicomponent intervention, it would not be possible to exclude changes related to other components of the trial in the follow-up analyses. Using data from the control group allowed us to use that group as a population in an observational follow-up design. Baseline characteristics are described by mean and standard deviation (SD) for continuous normally distributed variables, median and inter quartile range (IQR) for continuously non-normally distributed variables and number of patients and percentages for categorical variables.
To determine the effect of the COSMOS intervention on the five different sleep parameters (daytime sleep, total sleep time, sleep efficiency, wake after sleep onset, and early morning awakening), we performed separate linear mixed models analyses for each outcome measure, with fixed effects for group, time and their interaction (i.e., the intervention effect), and random intercepts for patients, and nursing home unit if necessary.

To investigate baseline associations between sleep parameters and antihypertensive medication use or blood pressure, fifteen separate linear regression models were fitted. We performed both unadjusted analyses, and analyses adjusted for age, gender and hypertensive diagnoses at baseline (yes/ no). Blood pressure measurements, both systolic and diastolic, were divided by 10 to increase the readability of the coefficients, a change of 1 in the coefficient reflects a $10-\mathrm{mmHg}$ change in blood pressure.

We used data from the control group to study associations between changes in sleep parameters and change in antihypertensive medication use or change in systolic or diastolic blood pressure from baseline to month 4 . The analyses were linear mixed effect models with fixed effects for exposure (antihypertensive medication use, systolic or diastolic pressure) and time, and random intercepts for patients, and nursing home unit if necessary. Results are reported both unadjusted and adjusted for age, gender and hypertensive diagnoses at baseline.

For the regression models, clustering was accounted for with regards to daytime sleep. For all other outcomes, clustering had no added effect to the model, and the simplest model was retained.

Significance level was set to 0.05 . Analyses were performed using STATA 16 (StataCorp, Texas, USA).

\section{Ethics, consent and permissions}

All patients and their families were informed about the study, both orally and in writing. The information described the intervention, what data would be collected, and that data collected would be published in scientific publications. When patients lacked the capacity to consent, informed presumed consent was obtained in writing from the family. If the patients in the actigraphy subproject appeared to be bothered by the actigraph, staff was instructed to help them remove the watch. The COSMOS trial adheres to the Helsinki declaration and Norwegian law and has been approved by the Regional Ethics Committee West (2013/1765). The trial is registered at clinicaltrials.gov (NCT02238652).

\section{Results}

Out of the 765 invited participants, the COSMOS trial included 545 patients from 67 nursing home units. The actigraphy subproject included 107 patients. Due to actigraph malfunction and missing data (less than 5 
recorded days and nights), 17 were excluded leaving 90 participants with actigraphy protocols who were eligible to be included in our analyses.

At baseline, $52 \% \quad(n=47)$ of the patients in the actigraph sub-group used antihypertensive medications. According to the diagnoses in the medical records, $2 \%$ $(n=2)$ of the patients had elevated blood pressure, and $27 \%(n=24)$ had uncomplicated hypertension (Table 1$)$. At month four, 11 patients in the intervention group and 5 in the control group had quitted using antihypertensive medications, while only one patient had started using these medications (from the intervention group).

Looking at both groups combined at baseline, we found no significant associations between sleep parameters and blood pressure or antihypertensive medication use (Table 2, systolic data shown).

Interestingly, looking at the intervention effects of the COSMOS study on sleep parameters, there was a significant reduction in total sleep time at month four in the intervention group as compared to the control group (mean difference in change $(\mathrm{MC})=-58.5 \mathrm{~min}, 95 \%$ Confidence Interval $(\mathrm{CI})=-115.5--1.6, p<0.05)$, with a corresponding reduction within the intervention group (42.5 $\mathrm{min}, \mathrm{CI}=-76.6--8.4, p<0.05)$. There were no other significant changes in sleep parameters from baseline to month four (Table 3).

Looking at the observational follow-up data in the control group, we found a significant association between increased systolic blood pressure and increased total sleep time from baseline to month 4 (MC $25.0 \mathrm{~min}$, $\mathrm{CI}=4.5-45.5, p<0.05)$ (Table 4$)$. There were significant negative associations between sleep onset latency and antihypertensive medication use ( $\mathrm{MC}-51.1 \mathrm{~min}, \mathrm{CI}=-$ $95.6--7.4, p<0.05)$ and systolic blood pressure (MC $-13.7 \mathrm{~min}, \mathrm{CI}=-22.0--5.4, p<0.05)$. Thus, antihypertensive medication use was associated with a shorter sleep onset latency. Similarly, increased systolic blood pressure was associated shorter sleep onset latency. Importantly, there was an association between antihypertensive medication use and increased daytime sleep within the control group from baseline to month 4 (MC $50.3 \mathrm{~min}$, $\mathrm{CI}=4.8-95.6, p<0.01)$. There were no significant associations between diastolic blood pressure and the sleep parameters. Changes in antihypertensive medication use and systolic pressure are presented in Tables 5 and 6 .

\section{Discussion}

Our primary aim was to investigate the association between antihypertensive medication use, blood pressure and day- and night-time sleep. Alongside our multicomponent intervention including a systematic medication review, we found a significant reduction in total sleep time at month 4 in the intervention group compared to the control group. Further, a significant association between increased systolic blood pressure and increased total sleep time were demonstrated, from baseline to month 4 in the control group. Both increasing blood pressure and antihypertensive medication use were associated with reduced sleep onset latency. Antihypertensive medication use was associated with increased daytime sleepiness. These findings are of key importance for the clinician because daytime sleep and nighttime sleep problems are common in nursing home patients of whom $50 \%$ are treated with antihypertensives despite almost $80 \%$ having low or normal blood pressure.

Over half of the patients in the actigraphy subgroup received antihypertensive medications, at baseline, which illustrates how common the use of such medications is in this population and thus also the clinical urgency of investigating potential side effects. Sleep problems have been related to severe health consequences; clinicians who prescribes and reviews the medications in this population should therefore consider the effects in this study carefully. The results also call for further investigation with a study design that includes sleep and antihypertensive medication use as the primary point of investigation.

We have previously demonstrated a significant higher deprescribing of antihypertensive medications in the intervention compared to the control group [28]. In that publication, also based on COSMOS data, the intervention group showed an increase in blood pressure when antihypertensive medications were reduced/withdrawn, from baseline to month four (see publication for full details on changes in medication and effects on blood pressure). It is possible that these changes also affected their sleep, although research is lacking to confirm this hypothesis. In addition, the medication review may have resulted in the discontinuation of several medications that impact sleep, such as antidepressants and hypnotics. Indeed, the reduced total sleep time in the intervention group compared to control may in part be a result of a withdrawal-effect. In our previous study on medication review, the blood pressure returned to baseline values from month four to month nine. Unfortunately, we have no actigraphy recordings for the nine-month follow-up, as it would be interesting to investigate whether the sleep improved in the intervention group in conjunction with the normalization of blood pressure.

It is also possible that changes in the clinical practice in the intervention units had an impact on the reduced total sleep time. In our education of the intervention units, we communicated that it may be disadvantageous to stay in bed longer than intended sleep time. Because actigraphy is sensitive to detect sleep but show less specificity for wakefulness, a substantially reduced time in bed could impact total sleep time. In order to investigate the longitudinal associations between antihypertensive 
Table 1 Baseline characteristics for nursing home residents in total, and by group

\begin{tabular}{|c|c|c|c|c|c|c|c|c|c|}
\hline & \multicolumn{3}{|c|}{ Control group } & \multicolumn{3}{|c|}{ Intervention group } & \multicolumn{3}{|c|}{ Both groups } \\
\hline & $\begin{array}{l}\text { Mean/ } \\
\mathrm{n} / \\
\text { median }\end{array}$ & $\begin{array}{l}(\mathrm{SD}) / \\
(\%) / \\
\left(\mathrm{IQR}^{\mathrm{a}}\right)\end{array}$ & $N$ & $\begin{array}{l}\text { Mean/ } \\
\text { n/ } \\
\text { median }\end{array}$ & $\begin{array}{l}(\mathrm{SD}) / \\
(\%) / \\
\left(\mathrm{IQR}^{\mathrm{a}}\right)\end{array}$ & $N$ & $\begin{array}{l}\text { Mean/ } \\
\mathrm{n} / \\
\text { median }\end{array}$ & $\begin{array}{l}(\mathrm{SD}) / \\
(\%) / \\
\left(\mathrm{IQR}^{\mathrm{a}}\right)\end{array}$ & $N$ \\
\hline \multicolumn{10}{|l|}{ Demographic variables: } \\
\hline Females & 26 & $(67 \%)$ & 39 & 43 & $(84 \%)$ & 51 & 69 & $(77 \%)$ & 90 \\
\hline Age & 85.3 & $(7.6)$ & 39 & 88.2 & $(8.3)$ & 51 & 87.0 & $(8.1)$ & 90 \\
\hline $\mathrm{BMI}$ & 23.5 & $(4.6)$ & 32 & 24.0 & (3.9) & 48 & 23.8 & $(4.2)$ & 80 \\
\hline Systolic blood pressure ${ }^{b}$ & 128 & $(17)$ & 37 & 130 & (18) & 49 & 129 & (18) & 86 \\
\hline Diastolic blood pressure ${ }^{b}$ & 70 & (13) & 37 & 70 & (12) & 49 & 70 & $(12)$ & 86 \\
\hline Activities of daily living $^{c}$ & 17.0 & $(5.5)$ & 39 & 17.8 & $(.15)$ & 51 & 17.3 & $(5.3)$ & 90 \\
\hline Regular prescriptions & 7.5 & $(4.0)$ & 39 & $7 . .6$ & (3.9) & 51 & 7.6 & (3.9) & 90 \\
\hline Diagnoses & 4.2 & $(2.0)$ & 39 & 4.5 & $(2.9)$ & 51 & 4.4 & $(2.6)$ & 90 \\
\hline \multicolumn{10}{|l|}{ Cognitive function: } \\
\hline $\operatorname{MMSE}^{\mathrm{a}}(0-30)$ & 12 & $(7-17)$ & 35 & 10 & $(5-13)$ & 49 & 11 & $(5-16.5)$ & 84 \\
\hline No $(24-30)$ & 2 & $(6 \%)$ & & 3 & $(6 \%)$ & & 5 & $(6 \%)$ & \\
\hline Mild (18-23) & 6 & $(17 \%)$ & & 7 & $(14 \%)$ & & 13 & $(15 \%)$ & \\
\hline Moderate (12-17) & 10 & $(28 \%)$ & & 10 & $(21 \%)$ & & 20 & $(24 \%)$ & \\
\hline Severe $(0-11)$ & 17 & $(49 \%)$ & & 29 & $(59 \%)$ & & 46 & $(55 \%)$ & \\
\hline Blood pressure $^{b}$ : & & & & & & & & & 86 \\
\hline Low (blood pressure < 120) & 12 & $(32 \%)$ & & 12 & $(24 \%)$ & & 24 & $(28 \%)$ & \\
\hline Normal $(120 \leq$ blood pressure $<141)$ & 18 & $(49 \%)$ & & 25 & $(52 \%)$ & & 43 & $(50 \%)$ & \\
\hline High (blood pressure $\geq 141$ ) & 7 & $(19 \%)$ & & 12 & $(24 \%)$ & & 19 & $(22 \%)$ & \\
\hline \multicolumn{10}{|l|}{ Hypertension diagnoses: } \\
\hline Elevated blood pressure (K85) & 1 & $(3 \%)$ & 39 & 1 & $(2 \%)$ & 51 & 2 & $(2 \%)$ & 90 \\
\hline Uncomplicated hypertension (K86) & 11 & $(28 \%)$ & 39 & 13 & $(25 \%)$ & 51 & 24 & $(27 \%)$ & 90 \\
\hline Hypertension with organ complications (K87) & 1 & $(3 \%)$ & 39 & 2 & $(4 \%)$ & 51 & 3 & $(3 \%)$ & 90 \\
\hline \multicolumn{10}{|l|}{ Medication (ATC Code): } \\
\hline Antihypertensive medications & 21 & $(54 \%)$ & 39 & 26 & $(51 \%)$ & 51 & 47 & $(52 \%)$ & 90 \\
\hline High-ceiling diuretics (CO3C) & 13 & $(33 \%)$ & 39 & 13 & $(25 \%)$ & 51 & 26 & $(29 \%)$ & 90 \\
\hline Beta-blockers (C07A) & 13 & $(33 \%)$ & 39 & 14 & $(27 \%)$ & 51 & 27 & $(30 \%)$ & 90 \\
\hline Plain angiotensin II antagonists (CO9C) & 3 & $(8 \%)$ & 39 & 9 & $(18 \%)$ & 51 & 12 & $(13 \%)$ & 90 \\
\hline Plain ACE inhibitors (C09A) & 3 & $(8 \%)$ & 39 & 2 & $(4 \%)$ & 51 & 5 & $(6 \%)$ & 90 \\
\hline $\begin{array}{l}\text { Calcium channel blockers, mainly vascular } \\
\text { effect (C08C) }\end{array}$ & 2 & $(5 \%)$ & 39 & 5 & $(10 \%)$ & 51 & 7 & $(8 \%)$ & 90 \\
\hline \multicolumn{10}{|l|}{ Sleep ${ }^{a}:$} \\
\hline Total sleep time (TST) & 443 & $(348-552)$ & 37 & 514 & $(425-601)$ & 46 & 492 & $(371-572)$ & 83 \\
\hline Sleep onset latency (SOL) & 24 & $(10-95)$ & 37 & 23 & $(10-64)$ & 46 & 24 & $(10-79)$ & 83 \\
\hline Wake after sleep onset & 149 & $(105-193)$ & 37 & 127 & $(82-202)$ & 46 & 142 & $(93-202)$ & 83 \\
\hline Daytime sleep (DTS) & 170 & $(102-237)$ & 37 & 115 & $(204-342)$ & 45 & 180 & $(110-276)$ & 82 \\
\hline Early morning awakening (EMA) & 39 & $(31-73)$ & 37 & 28 & $(14-56)$ & 46 & 35 & $(19-70)$ & 83 \\
\hline
\end{tabular}

Data are presented as mean (SD), number (\%) or median (IQR), with score ranges in parentheses

anter Quartile Range

${ }^{\mathrm{b}}$ Measured in $\mathrm{mmHg}$

${ }^{c}$ Measured on a scale from 0 to 30 , where 0 is independent and 30 is totally independent in activities of daily living

${ }^{\mathrm{d}}$ Higher scores indicate better cognitive function

${ }^{\mathrm{e}}$ Reported as average minutes per day/night 
Table 2 Associations between sleep parameters and blood pressure or antihypertensive medication use at baseline, for both groups combined

\begin{tabular}{|c|c|c|c|c|c|c|}
\hline Outcome $^{a}$ & Exposure & $\begin{array}{l}\text { Unadjusted association } \\
(95 \% \mathrm{Cl})\end{array}$ & $p$-value & $\begin{array}{l}\text { Adjusted association }{ }^{b} \\
(95 \% \mathrm{Cl})\end{array}$ & $p$-value & $\mathrm{N}$ \\
\hline Total sleep time & Anti hypertensives ${ }^{c}$ & $-1.4(-71.7-69.0)$ & 0.97 & $14.1(-63.7-91.9)$ & 0.72 & 83 \\
\hline Total sleep time & Systolic blood pressure ${ }^{d}$ & $-3.6(-24.2-17.1)$ & 0.73 & $-3.1(-24.9-18.7)$ & 0.78 & 79 \\
\hline Sleep onset latency & Anti hypertensives ${ }^{c}$ & $-11.6(-46.7-23.6)$ & 0.53 & $-20.9(-59.6-17.8)$ & 0.29 & 83 \\
\hline Sleep onset latency & Systolic blood pressure ${ }^{d}$ & $-0.9(-11.2-9.3)$ & 0.86 & $-2.4(-13.1-8.3)$ & 0.66 & 79 \\
\hline Wake after sleep onset & Anti hypertensives ${ }^{c}$ & $-14.9(-50.0-20.3)$ & 0.40 & $-12.0(-49.8-25.7)$ & 0.53 & 83 \\
\hline Wake after sleep onset & Systolic blood pressure ${ }^{d}$ & $4.5(-5.8-14.9)$ & 0.39 & $4.3(-6.2-14.7)$ & 0.42 & 79 \\
\hline Daytime sleep & Anti hypertensives ${ }^{c}$ & $22.7(-29.6-75.1)$ & 0.39 & $31.8(25.9-89.5)$ & 0.28 & 82 \\
\hline Daytime sleep & Systolic blood pressure ${ }^{d}$ & $-7.0(-22.0-8.2)$ & 0.36 & $-6.7(-22.7-7.4)$ & 0.41 & 78 \\
\hline Early morning awakening & Anti hypertensives ${ }^{c}$ & $-10.6(-39.8-18.6)$ & 0.47 & $-7.0(-38.8-24.8)$ & 0.67 & 83 \\
\hline Early morning awakening & Systolic blood pressure ${ }^{d}$ & $-4.9(-13.1-3.4)$ & 0.24 & $-3.9(-12.5-4.7)$ & 0.37 & 79 \\
\hline
\end{tabular}

${ }^{\mathrm{a}}$ Reported as average minutes per day/night

${ }^{\mathrm{b}}$ Adjusted for age, gender and hypertensive diagnoses at baseline (yes/ no)

cAnti-hypertensive users compared to non-anti-hypertensive users

${ }^{\mathrm{d}}$ Increase in sleep parameter associated with $10 \mathrm{mmHg}$ increase in systolic blood pressure

medication use and sleep without the contamination of our multicomponent intervention we also analysed the effects in the control group alone.

An important finding was the association between antihypertensive medication use and increased daytime sleep. This may indicate that the medication has a negative central nervous impact on these patients, affecting their wakefulness. Indeed, previous studies show that beta-blockers is associated with an increased incidence of daytime fatigue [20]. Fatigue and sleepiness are not identical symptoms, and the results may thus be interpreted slightly differently. It is possible that the antihypertensive medications led to increased lethargy and less movement. This would have been interpreted as sleep by an actigraphy. Alternatively, the medication use may have caused an increased propensity to sleep during the day. The latter, in particular would have affected the subsequent night-time sleep. Our group also investigated the association between low systolic blood pressure and neuropsychiatric symptoms in people with dementia using antihypertensive medication [33]. However, in this study, no difference between high and low systolic blood pressure and the symptom clusters were identified.

There was a negative association between sleep onset latency and antihypertensive medication use, which could be interpreted in relation to the daytime sleep findings. Daytime sleep is related to reduced build-up in sleep need, which is subsequently related to difficulties falling asleep and reduced deep sleep [44]. A downward spiral may thus be established, where extended daytime naps lead to difficulties falling asleep and poor nighttime sleep quality, which in turn increases the urge to nap during the day. Conversely, the significant association between sleep onset latency and high blood pressure, may be due to other mechanisms. It could have been expected that high blood pressure was related to a general higher level of arousal $[25,26]$. Since blood pressure were not related to daytime sleepiness, this could be the case, but the decreased sleep onset latency does not confirm this hypothesis. A study designed to review

Table 3 Estimated intervention effects, and changes within groups from baseline to month four, for five different sleep parameters

\begin{tabular}{|c|c|c|c|c|c|}
\hline \multirow[t]{3}{*}{ Outcome $^{a}$} & \multicolumn{2}{|c|}{ Within-group change } & \multirow{3}{*}{$\begin{array}{l}\text { Intervention } \\
\text { effect } \\
(95 \% \mathrm{Cl})\end{array}$} & \multirow[t]{3}{*}{$p$-value ${ }^{b}$} & \multirow[t]{3}{*}{$n$} \\
\hline & Control & Intervention & & & \\
\hline & $(95 \% \mathrm{Cl})$ & $(95 \% \mathrm{Cl})$ & & & \\
\hline Total sleep time & $16.0(-29.6-61.6)$ & $-42.5^{*}(-76.6--8.4)$ & $-58.5^{*}(-115.5--1.6)$ & 0.04 & 90 \\
\hline Sleep onset latency & $-7.2(-33.4-18.7)$ & $8.3(-11.2-27.9)$ & $15.6(-16.9-48.2)$ & 0.35 & 90 \\
\hline Wake after sleep onset & $2.9(-22.9-28.8)$ & $18.3(-1.1-37.7)$ & $15.4(-17.0-47.7)$ & 0.35 & 90 \\
\hline Daytime sleep & $10.6(-20.9-42.2)$ & $3.1(-20.7-27.0)$ & $-7.5(-47.1-32.0)$ & 0.71 & 90 \\
\hline Early morning awakening & $-24.3(-49.5-1.0)$ & $18.3(-1.1-37.7)$ & $28.6(-3.1-60.4)$ & 0.07 & 90 \\
\hline
\end{tabular}

${ }^{a}$ Reported as average minutes per day/night

${ }^{b} p$-value for intervention effect

${ }^{*} p<0.0$ 
Table 4 Associations between change in sleep parameters and change in blood pressure or change in antihypertensive medication use from baseline to month 4, in the control group

\begin{tabular}{|c|c|c|c|c|c|c|}
\hline Outcome $^{a}$ & Exposure & $\begin{array}{l}\text { Unadjusted association } \\
(95 \% \mathrm{Cl})\end{array}$ & $p$-value & $\begin{array}{l}\text { Adjusted association } \\
(95 \% \mathrm{Cl})\end{array}$ & $p$-value & $\mathrm{n}$ \\
\hline Total sleep time & Anti hypertensives ${ }^{c}$ & $15.6(-74.0-105.2)$ & 0.73 & $24.0(-71.5-119.5)$ & 0.62 & 39 \\
\hline Total sleep time & Systolic blood pressure ${ }^{d}$ & $23.5(3.2-43.8)$ & 0.02 & $25.0(4.5-45.5)$ & 0.02 & 38 \\
\hline Sleep onset latency & Anti hypertensives ${ }^{c}$ & $-44.8(-86.5--3.1)$ & 0.04 & $-51.1(-95.6--7.4)$ & 0.02 & 39 \\
\hline Sleep onset latency & Systolic blood pressure ${ }^{d}$ & $-12.7(-20.9--4.4)$ & 0.01 & $-13.7(-22.0--5.4)$ & 0.00 & 38 \\
\hline Wake after sleep onset & Anti hypertensives ${ }^{c}$ & $-8.7(-49.5-32.0)$ & 0.67 & $-12.7(-56.0-30.6)$ & 0.57 & 39 \\
\hline Wake after sleep onset & Systolic blood pressure ${ }^{d}$ & $-3.7(-13.9-6.5)$ & 0.48 & $-2.8(-13.1-7.6)$ & 0.60 & 38 \\
\hline Daytime sleep & Anti hypertensives ${ }^{c}$ & $61.0(4.3-117.8)$ & 0.035 & $50.3(4.8-95.6))$ & 0.03 & 39 \\
\hline Daytime sleep & Systolic blood pressure ${ }^{d}$ & $3.7(-8.4-15.8)$ & 0.55 & $1.9(-10.1-13.9)$ & 0.76 & 38 \\
\hline Early morning awakening & Anti hypertensive ${ }^{c}$ & $-20.4(-63.2-22.3)$ & 0.35 & $-13.1(-59.0-32.8)$ & 0.58 & 39 \\
\hline Early morning awakening & Systolic blood pressure ${ }^{d}$ & $-9.6(-20.2-1.0)$ & 0.08 & $-9.1(-19.9-1.7)$ & 0.1 & 38 \\
\hline
\end{tabular}

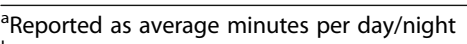

${ }^{\mathrm{b}}$ Adjusted for age, gender and hypertensive diagnoses at baseline (yes/ no)

'Anti-hypertensive users compared to non-anti-hypertensive users or change in anti-hypertensive medication use from baseline to month 4 for a patient

${ }^{d}$ Increase in sleep parameter associated with $10 \mathrm{mmHg}$ increase in systolic blood pressure, the association can be interpreted as between-patients association or

within-patient association

the effects of BP and antihypertensive medication use on central sleep parameters are needed to fully understand these effects.

There may be seasonal variations in sleeping patterns in nursing home patients. The COSMOS trial started the data collection during the summer, and the data collection continued throughout the following year, starting up in different municipalities in a stepwise manner. If all participants had started in the summer, the effect of the intervention on sleep could have been affected by the reduced light during winter. Meanwhile, with different startup times, seasonality is less likely to represent a bias in our dataset.

\section{Strengths and limitations}

There are some limitations to this study. We relied on the local methods for measuring blood pressure in each unit, which resulted in no standard procedure for assessment. Antihypertensive medications were often prescribed prior to nursing home placement; thus, we did not have reliable data on when and why antihypertensive medications were prescribed. The number of patients

Table 5 Changes in antihypertensive medication use from baseline to month 4, by group

\begin{tabular}{lll}
\hline Antihypertensive medication use & Control & Intervention \\
\hline Never used $^{\text {a }}$ & 18 & 24 \\
Starters $^{\mathrm{b}}$ & 0 & 1 \\
Quitters $^{\mathrm{c}}$ & 5 & 11 \\
Stable users $^{\mathrm{d}}$ & 16 & 15
\end{tabular}

${ }^{\mathrm{a}}$ Did not use at baseline or at month 4

${ }^{\mathrm{b}}$ Did not use at baseline, user at month 4

'User at baseline, but not at month 4

dUser at both baseline and month 4 who both used antihypertensive medications, and who were included in the actigraphy subprojects was low, and we cannot exclude the possibility of type 2 error. Our study population was representative for the nursing home population in general, i.e., polypharmacy was common with a mean use of over 7.6 regular medications. These medications often include medications such as antidepressants, opioids, and hypnotics which also affects sleep [45]. Thus, we cannot rule out the effects of other medications, or the discontinuation of these medications. Meanwhile, by investigation the longitudinal effects in the control group only, we excluded any effects related to the COSMOS medication review, and multicomponent intervention. Meanwhile, this observational design introduces an increased risk of confounding variables affecting the results. We did not correct for multiple testing in this study. We performed relatively few single variable regression analyses, including few variables in the same analysis, which could arguably make the need for correction less pressing. Together with the fact that we had a relatively low $\mathrm{n}$ in this study, the risk of a type 2 error would be high.

\section{Conclusions}

Antihypertensive medication use was frequent in our population of nursing home patients. Our results suggest a correlation between excessive daytime sleep and

Table 6 Changes in blood pressure from baseline to month 4, control group

\begin{tabular}{lll}
\hline & \multicolumn{2}{l}{ Control Mean (SD) } \\
\cline { 2 - 3 } & Baseline $\boldsymbol{N = 3 7}$ & Month $\mathbf{4} \mathbf{N = 2 7}$ \\
\hline Systolic pressure, $\mathrm{mmHg}$ & $128(17)$ & $123(16)$ \\
\hline
\end{tabular}


hypertensive medication use, which also can impact the night-time sleep. These findings are important, and needs to be further investigated, since antihypertensive medications are frequently used in nursing home patients, and sleep problems may be especially detrimental for this population.

\section{Abbreviations \\ ATC: Anatomical Therapeutic Chemical Index; BP: Blood Pressure; $\mathrm{Cl}$ : Confidence Interval; COSMOS: COmmunnication, Systematic pain assessment and treatment, Medication Review, Organization of activities, Security; IQR: Inter Quartile Range; MC: mean difference in change; RCT: Randomized Controlled Trial; SD: Standard Deviation; START 2: Screening Tool to Alert to Right Treatment; STOPP 2: Screening Tool of Older Persons' Prescriptions}

\section{Acknowledgements}

We would like to give a special thanks to all participating nursing homes including patients, staff and physicians who made this study possible. BSH would like to thank the G.C. Rieber Foundation and the Norwegian Government for supporting our work at the Centre for Elderly and Nursing Home Medicine, University of Bergen, Norway. Statistician Dagrun Slettebo at the Biostatistics and Data analysis core facility (BIOS), University of Bergen, carried out parts of the statistical analyses. We would also like to thank Irene Aasmul and Torstein Habiger for helping with the data collection.

\section{Authors' contributions}

$\mathrm{EF}, \mathrm{TE}, \mathrm{CG}$ and BSH made substantial contributions to the conception and design of the work. EF, TE, CG and BSH made substantial contributions to the data acquisition, EF, CG and BSH made substantial contributions to the analysis, and EF, TE, CG and BSH made substantial contributions to the interpretation of data. EF have drafted the work, while TE, CG and BSH have substantively revised it. EF, TE, CG and BSH have all approved the submitted version and have agreed both to be personally accountable for own contributions and to ensure that questions related to the accuracy or integrity of any part of the work, even ones in which the author was not personally involved, are appropriately investigated, resolved, and the resolution documented in the literature.

\section{Funding}

This study was sponsored by the Norwegian Research Council (Sponsor's Protocol Code: 222113/H10), the Rieber foundation and the Rebekka Ege Hegerman' trust. The sponsors had no say in design, execution, data collection, analyses, or writing of the paper.

\section{Availability of data and materials}

The dataset analysed during the current study are not publicly available due restrictions made by the ethical committee for safe data storage, but may be available upon reasonable request made to the corresponding author.

\section{Declarations}

\section{Ethics approval and consent to participate}

All patients and their families were informed about the study, both orally and in writing. When patients lacked the capacity to consent, informed presumed consent was obtained in writing from the family. If the patients in the actigraphy subproject appeared to be bothered by the actigraph, staff was instructed to help them remove the watch. The COSMOS trial adheres to the Helsinki declaration and Norwegian law and has been approved by the Regional Ethics Committee West (2013/1765). The trial is registered at clinicaltrials.gov (NCT02238652).

\section{Consent for publication}

Not applicable.

\section{Competing interests}

The authors declare that they have no competing interests.

\section{Author details}

${ }^{1}$ Centre for Elderly and Nursing Home Medicine, Department of Global Public Health and Primary Care, University of Bergen, Bergen, Norway. ${ }^{2}$ Department of Clinical Psychology, University of Bergen, Postboks 7807, 5020 Bergen, Norway. ${ }^{3}$ National Centre for Emergency Primary Health Care, NORCE Norwegian Research Centre, Bergen, Norway. ${ }^{4}$ Municipality of Bergen, Bergen, Norway.

Received: 19 November 2020 Accepted: 6 June 2021

Published online: 18 July 2021

\section{References}

1. Bliwise DL. Sleep disorders in Alzheimer's disease and other dementias. Clin Cornerstone. 2004;6(1):16-28.

2. Fetveit A, Bjorvatn B. Bright-light treatment reduces actigraphic-measured daytime sleep in nursing home patients with dementia: a pilot study. Am J Geriatr Psychiatry. 2005;13(5):420-3.

3. Jacobs D, Ancoli-Israel S, Parker L, Kripke DF. Twenty-four-hour sleep-wake patterns in a nursing home population. Psychol Aging. 1989;4(3):352.

4. Fetveit A, Bjorvatn B. Sleep duration during the 24-hour day is associated with the severity of dementia in nursing home patients. Int J Geriatr Psychiatry. 2006;21(10):945-50.

5. Gordon AL, Franklin M, Bradshaw L, Logan P, Elliott R, Gladman JR. Health status of UK care home residents: a cohort study. Age Ageing. 2014;43(1): 97-103

6. Mancia G, Fagard R, Narkiewicz K, Redon J, Zanchetti A, Bohm M, et al. 2013 ESH/ESC guidelines for the management of arterial hypertension: the Task Force for the Management of Arterial Hypertension of the European Society of Hypertension (ESH) and of the European Society of Cardiology (ESC). Eur Heart J. 2013;34(28):2159-219.

7. Bejan-Angoulvant T, Saadatian-Elahi M, Wright JM, Schron EB, Lindholm LH, Fagard $\mathrm{R}$, et al. Treatment of hypertension in patients 80 years and older: the lower the better? A meta-analysis of randomized controlled trials. J Hypertens. 2010;28(7):1366-72.

8. Supiano MA, Williamson JD. Applying the Systolic Blood Pressure Intervention Trial Results to Older Adults. J Am Geriatr Soc. 2017;65(1):16-21.

9. McNeil MJ, Kamal AH, Kutner JS, Ritchie CS, Abernethy AP. The Burden of Polypharmacy in Patients Near the End of Life. J Pain Symptom Manage. 2016:51(2):178 - 83.e2

10. Mangoni AA, Jackson SH. Age-related changes in pharmacokinetics and pharmacodynamics: basic principles and practical applications. $\mathrm{Br} J \mathrm{Clin}$ Pharmacol. 2004;57(1):6-14.

11. Harrison JK, Van Der Wardt V, Conroy SP, Stott DJ, Dening T, Gordon AL, et al. New horizons: the management of hypertension in people with dementia. Age Ageing. 2016;45(6):740-6.

12. Betts T, Alford C. $\beta$-Blocking Drugs and Sleep A Controlled Trial. Drugs. 1983;25(2):268-72.

13. Gislason T, Almqvist M. Somatic diseases and sleep complaints. An epidemiological study of 3,201 Swedish men. Acta Med Scand. 1987;221(5): 475-81.

14. Nicholson AN, Roberts DP, Stone BM, Turner C. Antihypertensive therapy in critical occupations: studies with an angiotensin II antagonist. Aviat Space Environ Med. 2001;72(12):1096-101.

15. van der Wardt V, Logan P, Conroy S, Harwood R, Gladman J. Antihypertensive Treatment in People With Dementia. J Am Med Dir Assoc. 2014;15(9):620-9.

16. Zuurbier LA, Luik Al, Leening MJ, Hofman A, Freak-Poli R, Franco OH, et al. Associations of heart failure with sleep quality: the Rotterdam Study. J Clin Sleep Med. 2015;11(2):117-21.

17. Eshkoor SA, Hamid TA, Nudin SS, Mun CY. Importance of hypertension and social isolation in causing sleep disruption in dementia. Am J Alzheimer's Dis Other dement. 2014;29(1):61-6.

18. Brassington GS, King AC, Bliwise DL. Sleep problems as a risk factor for falls in a sample of community-dwelling adults aged 64-99 years. J Am Geriatr Soc. 2000;48(10):1234-40.

19. Suzuki R, Meguro M, Meguro K. Sleep disturbance is associated with decreased daily activity and impaired nocturnal reduction of blood pressure in dementia patients. Arch Gerontol Geriatr. 2011;53(3):323-7.

20. Kostis JB, Rosen RC, Holzer BC, Randolph C, Taska LS, Miller MH. CNS side effects of centrally-active antihypertensive agents: a prospective, placebo- 
controlled study of sleep, mood state, and cognitive and sexual function in hypertensive males. Psychopharmacology. 1990;102(2):163-70.

21. Ohayon MM, Carskadon MA, Guilleminault C, Vitiello MV. Meta-analysis of quantitative sleep parameters from childhood to old age in healthy individuals: developing normative sleep values across the human lifespan. Sleep. 2004;27(7):1255-73.

22. Flo E, Bjorvatn B, Corbett A, Pallesen S, Husebo BS. Joint occurrence of pain and sleep disturbances in people with dementia. A systematic review. Curr Alzheimers Res. 2016;14:538-45.

23. Parati G, Lombardi C, Castagna F, Mattaliano P, Filardi PP, Agostoni P. Heart failure and sleep disorders. Nat Rev Cardiol. 2016;13(7):389-403.

24. Foley DJ, Monjan A, Simonsick EM, Wallace RB, Blazer DG. Incidence and remission of insomnia among elderly adults: an epidemiologic study of 6,800 persons over three years. Sleep. 1999;22(Suppl 2):366-72.

25. Vargas I, Nguyen AM, Muench A, Bastien CH, Ellis JG, Perlis ML. Acute and Chronic Insomnia: What Has Time and/or Hyperarousal Got to Do with It? Brain Sciences. 2020;10(2):71.

26. Li Y, Vgontzas AN, Fernandez-Mendoza J, Bixler EO, Sun Y, Zhou J, et al. Insomnia with physiological hyperarousal is associated with hypertension. Hypertension. 2015;65(3):644-50.

27. Scheer FA, Morris CJ, Garcia JI, Smales C, Kelly EE, Marks J, et al. Repeated melatonin supplementation improves sleep in hypertensive patients treated with beta-blockers: a randomized controlled trial. Sleep. 2012;35(10):1395-402.

28. Gulla C, Flo E, Kjome RL, Husebo BS. Deprescribing antihypertensive treatment in nursing home patients and the effect on blood pressure. Journal of geriatric cardiology: JGC. 2018;15(4):275-83.

29. Husebo BS, Ballard C, Aarsland D, Gulla C, Aasmul I, Elvegaard T, et al. The Effect of a Multicomponent Intervention on Quality of Life in Residents of Nursing Homes: A Randomized Controlled Trial (COSMOS). J Am Med Direct Assoc. 2018;20(3):330-9.

30. Husebo BS, Flo E, Aarsland D, Selbaek G, Testad I, Gulla C, et al. COSMOSimproving the quality of life in nursing home patients: protocol for an effectiveness-implementation cluster randomized clinical hybrid trial. Implement Sci. 2015;10(1):131.

31. Aasmul I, Husebo BS, Flo E. Description of an advance care planning intervention in nursing homes: outcomes of the process evaluation. BMC Geriatr. 2018;18(1):26.

32. Aasmul I, Husebo BS, Sampson EL, Flo E. Advance Care Planning in Nursing homes-Improving the Communication between Patient, Family and Staff: Results from a Cluster. Randomized Controlled Trial. Front Psychol. 2018;9: 2284.

33. de Jong-Schmit BEM, Poortvliet RKE, Böhringer S, Bogaerts JMK, Achterberg WP, Husebo BS. Blood pressure, antihypertensive medication and neuropsychiatric symptoms in older people with dementia: The COSMOS study. Int J Geriatr Psychiatry. 2021;36(1):e5388.

34. Orfaly RA, Frances JC, Campbell P, Whittemore B, Joly B, Koh H. Train-thetrainer as an educational model in public health preparedness. J Public Health Manage Pract. 2005;Suppl:S123-7. https://doi.org/10.1097/00124 784-200511001-00021.

35. Guidelines for medication reviews. Norwegian Directorate of Health. Oslo, Norway: Norwegian Directorate of Health; 2012.

36. Agency NM. Check list for medication review. 2014.

37. O'Mahony D, O'Sullivan D, Byrne S, O'Connor MN, Ryan C, Gallagher P. STOPP/START criteria for potentially inappropriate prescribing in older people: version 2. Age Ageing. 2015;44(2):213-8.

38. Duran CE, Azermai M, Vander Stichele RH. Systematic review of anticholinergic risk scales in older adults. Eur J Clin Pharmacol. 2013;69(7): $1485-96$.

39. Van Remoortel H, Raste Y, Louvaris Z, Giavedoni S, Burtin C, Langer D, et al. Validity of six activity monitors in chronic obstructive pulmonary disease: a comparison with indirect calorimetry. PLoS One. 2012;7(6):e39198.

40. Lambiase MJ, Gabriel KP, Chang YF, Kuller LH, Matthews KA. Utility of actiwatch sleep monitor to assess waking movement behavior in older women. Med Sci sports Exerc. 2014;46(12):2301-7.

41. ATC/DDD Index. WHO Collaborating Centre for Drug Statistics Methodology. 2015. Available from: http://www.whocc.no/atc_ddd_index/.

42. Folstein MF, Folstein SE, McHugh PR. Mini-Mental State: Practical method for grading cognitive state of patients for clinician. J Psychiatr Res. 1975;12:189-98
43. Lawton MP, Brody EM. Assessment of Older People - Self-Maintaining and Instrumental Activities of Daily Living. Gerontologist. 1969;9:179-8.

44. Cooke JR, Ancoli-Israel S Normal and Abnormal Sleep in the Elderly. Handbook of clinical neurology / edited by PJ Vinken and GW Bruyn. 2011; 98:653- 65 .

45. Gulla C, Flo E, Kjome RLS, Husebo BS. Implementing a novel strategy for interprofessional medication review using collegial mentoring and systematic clinical evaluation in nursing homes (COSMOS). BMC Geriatr. 2019;19(1):130.

\section{Publisher's Note}

Springer Nature remains neutral with regard to jurisdictional claims in published maps and institutional affiliations.
Ready to submit your research? Choose BMC and benefit from:

- fast, convenient online submission

- thorough peer review by experienced researchers in your field

- rapid publication on acceptance

- support for research data, including large and complex data types

- gold Open Access which fosters wider collaboration and increased citations

- maximum visibility for your research: over $100 \mathrm{M}$ website views per year

At BMC, research is always in progress.

Learn more biomedcentral.com/submissions 